\title{
Corneal stromal demarcation line after collagen cross-linking in corneal ectatic diseases: a review of the literature
}

This article was published in the following Dove Press journal:

Clinical Ophthalmology

19 September 2016

Number of times this article has been viewed

\section{Leopoldo Spadea \\ Emanuele Tonti \\ Enzo Maria Vingolo}

Department of Medico-Surgical Sciences and Biotechnologies, Sapienza University of Rome, Latina, Italy
Correspondence: Leopoldo Spadea Via Benozzo Gozzoli 34, 00I42

Rome, Italy

Tel +3965193220

$\mathrm{Fax}+39688657818$

Email leopoldo.spadea@uniromal.it
Abstract: Collagen cross-linking (CXL) is a relatively new conservative approach for progressive corneal ectasia, which is able to strengthen corneal tissue reforming new covalent bonds. Subjective and objective results following this method seem to be promising. In recent years, newer CXL protocols have been developed to perform more effective and less invasive procedures. The increasing diffusion of CXL in the corneal ectatic disease has increased the need to have actual indices regarding the efficacy of the treatment. Evaluation of demarcation line (DL), a transition zone between the cross-linked anterior corneal stroma and the untreated posterior corneal stroma, is considered a measurement of the depth of CXL treatment into the stroma. Some evidence in the literature emphasize that DL could be a measure of effectiveness of the CXL. On the contrary, some authors believe that the "the deeper, the better" principle is rather a simplistic approach for interpreting the clinical importance of the corneal stromal DL.

Keywords: corneal cross-linking, demarcation line, keratoconus, ultraviolet A

\section{Introduction}

Keratoconus is one of the leading causes of cornea transplant. Therapies based on the spectacles and contact lenses do not hinder the progression of the disease. Surgeries and corneal transplantation, though preserved as the first choice for the severe variants of the disease, have risks such as rejection of the transplanted cornea. Corneal crosslinking $(\mathrm{CXL})$ is a noble strategy based on the underlying pathology of the disease. The main indication for CXL is to slow the progression of corneal ectatic diseases, such as keratoconus, pellucid marginal degeneration (PMD), and keratoglobus. ${ }^{1,2}$ In addition to these primary forms, there also exist forms related to refractive surgery. ${ }^{3}$

Studies on keratoconus epidemiology reported an incidence of 1.3-22.3 cases per 100,000 and a prevalence of $0.4-86$ cases per $100,000.4$ The incidence of keratectasia after laser in situ keratomileusis (LASIK) has been reported to be $0.04 \%-0.6 \%{ }^{5-7}$ Keratoconus is a progressive noninflammatory disease of the cornea distinguished by para-central corneal thinning and ectasia, resulting in irregular astigmatism with impaired vision. In most cases, it is bilateral but often asymmetrical and it usually begins sometime in puberty and it progresses until the fourth decade, when the corneal shape generally becomes stable. ${ }^{8}$ The etiology of keratoconus is unknown including genetic, biochemical, and physical factors. There is no unique theory elucidating its range of clinical appearances, and it is probable that keratoconus is the manifestation for several different conditions. It usually appears as an isolated disease, but it has been associated with a number of ocular and systemic pathologies, including vernal disease, 
retinitis pigmentosa, blue sclera, atopy, magnesium deficiency, Down's syndrome, Turner syndrome, and connective tissue disorders such as Marfan's syndrome, Ehlers-Danlos syndrome, osteogenesis imperfecta, and pseudoxanthoma elasticum. ${ }^{9}$ Genetic factors have an incidence of up to $10 \% .{ }^{10}$ In keratoconus corneas, the protease enzymes are upregulated while the activities of protease inhibitors are inhibited, involving an increasing digestion of stromal proteins with a consequent reduction in the biomechanical stability and corneal thinning. ${ }^{11,12}$ In diabetic patients, the severity of the pathology is inversely proportional. ${ }^{13}$ Corneal alterations in keratoconus involve changes in basement membrane structure, stromal collagen fiber, morphology of keratocytes, and abnormal cell-matrix interactions. ${ }^{14}$

Posterior keratoconus is a rare corneal anomaly, which is characterized by a total or localized noninflammatory thinning of the posterior stroma and a depression of the posterior corneal surface, whereas the anterior surface remains more regular. ${ }^{15}$

Less common than keratoconus is PMD, which usually affects the para-central and the inferior peripheral cornea in $\sim 85 \%$ of cases, while the superior peripheral cornea in remaining $15 \%$. It is a progressive noninflammatory disorder characterized by a band of thinning that usually involves the cornea between 4 o'clock and 8 o'clock positions, ie, $1 \mathrm{~mm}$ from the limbus. The etiology of PMD is not known. Corneal topography shows the typical "butterfly pattern" of an irregular astigmatism with a marked flattening along a vertical axis and a steepening of the inferior cornea., ${ }^{2,16}$

\section{Corneal CXL}

Corneal CXL with riboflavin and ultraviolet A (UV-A) is a technique to strengthen corneal tissue using riboflavin as a photosensitizer and UV-A to increase the formation of intra- and interfibrillar covalent bonds by photosensitized oxidation. This technique is able to flatten corneal steepening, stabilize the cornea, and reduce the refractive error improving the irregular astigmatism. ${ }^{17}$ Several studies confirm the effectiveness and safety of conventional cross-linking procedure, which is also known as "Dresden protocol", ${ }^{18-21}$ in which the interaction between $0.1 \%$ riboflavin molecules absorbed in corneal tissue and UV-A rays delivered at $3 \mathrm{~mW} / \mathrm{cm}^{2}$ for 30 minutes $\left(5.4 \mathrm{~J} / \mathrm{cm}^{2}\right.$ energy dose) releases reactive oxygen species that promote the formation of "molecular bridges" between and within collagen fibers. ${ }^{17,22}$

Riboflavin (vitamin B2), because of its alkylisoalloxazine structure, allows the absorption of a wide range of light spectrum, including an absorption peak in UV-A range, so it is the standard photoinducer in cross-linking. An effective cross-linking requires a considerable corneal absorption of riboflavin; therefore, the epithelium debridement in standard protocols (epithelium-off [epi-off] CXL) has the goal of overcoming the barrier formed by corneal epithelium tight junctions that would limit the penetration of macromolecule as vitamin B2 (molecular weight: $376.37 \mathrm{~g} / \mathrm{mol}$ ). ${ }^{23}$ However, the epithelium debridement is not free from complications, where there is an increased risk of ulcers, infections, haze, scarring, infiltrates, longer recovery time, patient discomfort, and postoperative pain. ${ }^{24}$ Thus, some effort has been put into the development of transepithelial (epithelium-on, epi-on) CXL. Since riboflavin cannot penetrate intact corneal epithelium due to its chemical properties, different strategies were studied to enhance transepithelial riboflavin penetration, such as increasing riboflavin imbibition time and new riboflavin solution formulations to facilitate its transepithelial penetration.

Therefore, some substances, such as benzalkonium chloride, ethylenediaminetetraacetic acid, and trometamol, were used to enhance the epithelial penetration of riboflavin with good results, allowing a transepithelial CXL. This technique was safe and effective especially in ultrathin keratoconic corneas (pachymetry $<400 \mu \mathrm{m}$ ), to avoid CXL cytotoxic effect on endothelium, crystalline lens, and other intraocular tissue. ${ }^{25}$ In fact, in transepithelial CXL, the epithelium acts as a UV filter because of the high absorption coefficient; in the absence of a photosensitizer, UV-A light at wavelengths of $350 \mathrm{~nm}$ and irradiance of $3 \mathrm{~mW} / \mathrm{cm}^{2}$ would not cause significant cytotoxicity. The addition of riboflavin reduces the damage threshold compared with UV-A light alone and produces oxygen-free radicals inducing CXL.

In recent years, to perform less invasive procedures, attention has focused on the possibility to create new protocols to minimize UV exposure duration, reduce postoperative discomfort, and further decrease the complication rate. ${ }^{3}$ In this direction, the accelerated, high-fluence CXL arises, based on the principle of photochemical reciprocity, known as the law of Bunsen-Roscoe, which states that in a system the same photochemical effect can be achieved through reduced irradiation interval while maintaining a constant total energy level by an increase in irradiation intensity. ${ }^{26,27}$

Ocular iontophoresis is a noninvasive protocol that uses the ions flow in an electric field to apply the negatively charged riboflavin within the stroma through the corneal epithelial barrier. In the most recent CXL protocols, it is a well-known method investigated to enhance the penetration of photosensitizer in the stroma. It has been evidenced that 
by using iontophoresis, a 5-minute imbibition achieves a sufficient riboflavin concentration in the corneal stroma for CXL treatment, preserving epithelial integrity. ${ }^{28}$ Several ex vivo studies confirmed the effectiveness of iontophoresis imbibition in obtaining an adequate riboflavin concentration into the stroma and the induction of evident biomolecular and structural changes of the treated corneas. ${ }^{29}$ To reduce the administration time, UV-A irradiation protocols that are different from the standard technique ( $3 \mathrm{~mW} / \mathrm{cm}^{2}$ for 30 minutes) can be used. Literature shows, in ex vivo experiments, a similar increase in corneal biomechanical stiffness using $10 \mathrm{~mW} / \mathrm{cm}^{2}$ illumination intensity for 9 minutes and the standard protocol ( $3 \mathrm{~mW} / \mathrm{cm}^{2}$ for 30 minutes). ${ }^{30}$ Preliminary clinical results of iontophoresis-assisted transepithelial (I-CXL) are very promising, reducing or blocking the progression of keratoconus without significant complications. ${ }^{31,32}$

\section{CXL combined with other treatments}

Corneal cross-linking has been used in combination with other treatments in keratoconic eyes and post-laser ectasia to optimize visual outcomes. Combined CXL and limited topography-guided photorefractive keratectomy in selected eyes with moderate ectasia and adequate corneal thicknesses have been evidenced to be effective with improvements in visual, refractive, and topographic data and stabilization of the ectatic disease in many eyes. ${ }^{33-35}$ Such different treatment modalities have been reported to be associated with significant improvements in the quality of life. However, the follow-up in these studies is limited to 1-3 years so that the long-term results are not known. In addition, significant corneal haze/scarring has been reported. ${ }^{36} \mathrm{CXL}$ has also been combined with intracorneal ring segment insertion, suggesting a potential additional effect of CXL. ${ }^{37,38}$ The transepithelial iontophoresis CXL has also been used in the treatment of PMD associated with sliding keratoplasty, proving to be safe and effective in the treatment of advanced PMD. ${ }^{39}$

CXL has also been associated with refractive procedures such as photorefractive keratectomy and LASIK to improve the long-term stability and to reduce the possible occurrence of postoperative ectasia. Up to now, such studies are very limited in terms of eyes treated, efficacy, and long-term follow-up, although a study in CXL after hyperopic LASIK demonstrated less regression of refractive effects over the follow-up period. ${ }^{40}$

\section{Demarcation line}

After corneal CXL in keratoconic eyes, some confocal microscopic studies evidenced edema, rarefaction of keratocytes in the anterior and mid-stroma, nerve loss, and focal endothelial changes in the immediate postoperative period (Table 1). ${ }^{41-43}$ Ultrastructural study shows that changes in stromal keratocytes with cell and collagen fiber shrinkage, chromatin condensation, and apoptotic bodies are detectable within 60 minutes after UV-A-riboflavin treatment. ${ }^{44}$ In the excited state, post UV-A exposure, riboflavin can produce radicals or singlet oxygen molecules that induce covalent bonds that can connect one polymer chain to another, causing in vivo cornea links in collagen fibers. ${ }^{45}$ Photochemically induced cross-links in cornea can be detected through structural changes secondary to CXL. Ex vivo studies evidence an increased resistance to enzymatic degradation processes involved in progressive thinning of the cornea in keratoconus. Corneas that underwent CXL treatment present an inferior rate of enzymatic collagenasic degradation when compared with an untreated cornea. Corneal CXL acts unevenly within the corneal thickness; UV-A intensity is inversely proportional to deepening in stromal tissue for several reasons;

Table I Comparative findings of corneal stromal DL depth after CXL

\begin{tabular}{|c|c|c|c|c|c|}
\hline Study & Patients (N) & CXL protocol & DL depth & Detection modality & $\begin{array}{l}\text { Time of DL } \\
\text { detection }\end{array}$ \\
\hline Seiler and Hafezi ${ }^{47}$ & 16 & Conventional & $300 \mu \mathrm{m}$ & Confocal microscopy & 2 weeks \\
\hline Doors et $\mathrm{al}^{51}$ & 28 & Conventional & $313 \mu \mathrm{m}$ & AS-OCT & I month \\
\hline Yam et $\mathrm{al}^{49}$ & 40 & Conventional & $28 \mathrm{I} \mu \mathrm{m}$ & AS-OCT & 6 months \\
\hline Filippello et $\mathrm{al}^{61}$ & 20 & Transepithelial & $100 \mu \mathrm{m}$ & AS-OCT & 2 weeks \\
\hline Kymionis et $\mathrm{al}^{56}$ & 16 & Conventional vs accelerated & $35 \mathrm{I} \mu \mathrm{m} ; 288 \mu \mathrm{m}$ & AS-OCT & I month \\
\hline Mazzotta et al ${ }^{59}$ & 20 & Accelerated $\mathrm{PL}$ vs $\mathrm{CL}$ & $215 \mu \mathrm{m}$ PL; $160 \mu \mathrm{m} \mathrm{CL}$ & $\begin{array}{l}\text { AS-OCT/confocal } \\
\text { microscopy }\end{array}$ & I month \\
\hline Moramarco et al ${ }^{50}$ & 60 & Accelerated PL vs CL & $213 \mu \mathrm{m}$ PL; $149 \mu \mathrm{m} \mathrm{CL}$ & AS-OCT & I month \\
\hline Bonnel et $\mathrm{al}^{64}$ & 12 & lontophoresis & $247 \mu \mathrm{m}$ & AS-OCT & I month \\
\hline Kymionis et $\mathrm{al}^{48}$ & 29 & Conventional vs accelerated & $342 \mu \mathrm{m} ; 3$ I $3 \mu \mathrm{m}$ & AS-OCT & I month \\
\hline Bikbova and Bikbov 65 & 119 & Conventional vs iontophoresis & $292 \mu \mathrm{m} ; \mathrm{I} 72 \mu \mathrm{m}$ & AS-OCT & 2 weeks \\
\hline
\end{tabular}

Abbreviations: AS-OCT, anterior segment optical coherence tomography; CL, continuous light; CXL, collagen cross-linking; DL, demarcation line; PL, pulsed light. 
and primarily riboflavin concentration decreases linearly with increasing of corneal depth, in accordance with diffusion gradient. Furthermore, according to Lambert-Beer law, $65 \%$ of UV radiation is absorbed in the first $200 \mu \mathrm{m}$. The consequence of a higher UV intensity in anterior cornea is a greater stiffening effect within first $250 \mu \mathrm{m}{ }^{28}$

Corneal cross-linking causes a dose-dependent keratocytes damage. Wollensak et $\mathrm{al}^{46}$ described cellular apoptosis to a depth of $300 \mu \mathrm{m}$ radiating with UV-A at $3 \mathrm{~mW} / \mathrm{cm}^{2}$. Histopathological studies showed an already complete keratocyte apoptosis limited to the anterior stroma within 24 hours. $^{46}$ An increased density of the extracellular matrix was evidenced to a depth of 300-350 $\mu \mathrm{m}$. By using the slit-lamp examination, this appears as a "demarcation line" (DL), a transition zone between the cross-linked stroma and untreated tissue, which can be seen as early as 2 weeks (Figure 1). It is an area of hyper-reflectivity due to different refractive indices or reflective properties of cross-linked vs untreated corneal stroma. Some authors characterized the corneal stromal DL as a clinical sign to evaluate the depth of the CXL treatment. ${ }^{47,48}$

Some studies hypothesize the role of the DL after CXL depth as representative of CXL effectiveness. ${ }^{49-51}$ Recently, the essential debate focused on whether the depth of the corneal stromal DL is indeed a true indicator of CXL efficacy. The main question is whether "the deeper, the better" principle can be applied to CXL. Actually, other confounding factors may interfere with the clinical interpretation of the depth of corneal DL after CXL. Therefore, some authors believe that the "the deeper, the better" principle is rather a

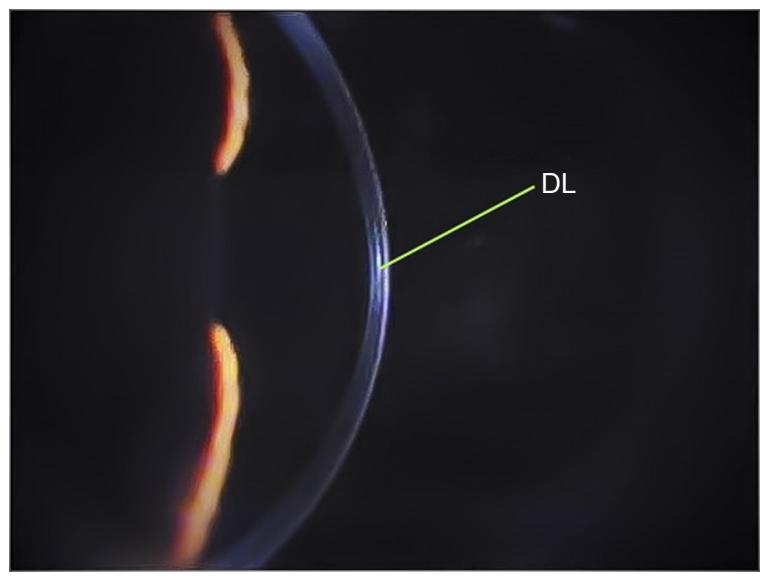

Figure I Biomicroscopic image of keratoconus eye at slit lamp 6 months after epi-off standard corneal CXL.

Note: $\mathrm{DL}$ is visible in the middle of the cornea.

Abbreviations: CXL, collagen cross-linking; DL, demarcation line; epi-off, epithelium-off.

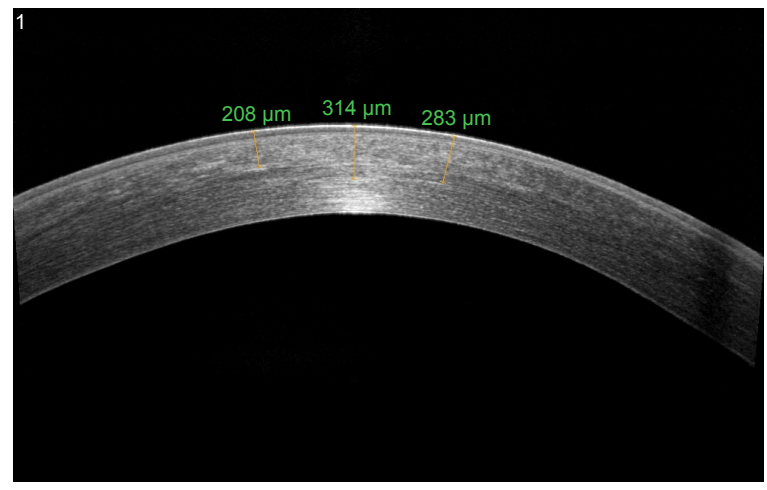

Figure 2 AS-OCT of keratoconus eye 6 months after epi-off corneal cross-linking radiating with UV-A at $3 \mathrm{~mW} / \mathrm{cm}^{2}$.

Note: $\mathrm{DL}$ is visible to a depth of $314 \mu \mathrm{m}$.

Abbreviations: AS-OCT, anterior segment optical coherence tomography; DL, demarcation line; epi-off, epithelium-off; UV-A, ultraviolet A.

simplistic approach for interpreting the clinical importance of the corneal stromal DL. ${ }^{52}$

In recent years, anterior segment optical coherence tomography (AS-OCT) and confocal microscopy have been used as tools to assess the depth of DL and consequently the depth of the cross-linking effect (Figure 2). Kymionis et al ${ }^{53}$ compared the depth of stromal DL using both AS-OCT and confocal microscopy studying the transition area between the cellular and acellular zone, thereby showing no statistical significant differences between the two measurements.

By using the AS-OCT, the stromal DL is detected within an enhanced image of the cornea in the horizontal meridian. The image is captured when the corneal reflex is visible, and the depth of DL is measured using the caliper tool provided by the manufacturer.

Doors et al described the best visibility of corneal stromal DL using AS-OCT at 1 month after CXL treatment, with an average DL depth of $313 \mu \mathrm{m}$; Yam et al measured the depth of DL at 6 months highlighting that the severity of ectasia and age may cause a worse DL visibility. ${ }^{49,51}$

A characteristic DL depth pattern, deeper centrally than peripherally has been observed in different studies; this is probably due to the top-hat beam profile of UV-A optical system that does not compensate for natural corneal curvature, so the UV-A beam enters the cornea at a non-orthogonal angle in the corneal periphery (Figure 2). ${ }^{54}$

\section{DL in accelerated epi-off $C X L$}

Corneal stromal DL after CXL using both the standard Dresden protocol (30 minutes with $3 \mathrm{~mW} / \mathrm{cm}^{2}$ ) and a modified accelerated protocol (14 minutes with $\left.9 \mathrm{~mW} / \mathrm{cm}^{2}\right)$ was evaluated according to the photochemical law of reciprocity (Bunsen-Roscoe law). There was no statistically significant 
difference in corneal stromal DL depth between the two groups, with a mean depth of $337 \mu \mathrm{m}$ in the first group and $322 \mu \mathrm{m}$ in the second group. ${ }^{55}$ However, Kymionis et al evaluated the corneal stromal DL depth after very highintensity $\left(18 \mathrm{~mW} / \mathrm{cm}^{2}\right) \mathrm{UV}$-A irradiation for a 5 -minute collagen CXL. Despite the easy identification of DL using AS-OCT, it seemed to be shallower when compared with the previously described 10-minute protocol and standard Dresden protocol (Figure 3). ${ }^{48,56}$

This confirms Shetty et al' $\mathrm{s}^{57}$ findings comparing the clinical effect and DL using four different protocols; they have noticed the presence of a DL at a depth of $280 \pm 47 \mathrm{~mm}$ in $3 \mathrm{~mW} / \mathrm{cm}^{2}$ irradiation for the 30 -minute group, $292 \pm 73 \mu \mathrm{m}$ in $9 \mathrm{~mW} / \mathrm{cm}^{2}$ for the 10-minute group, $203 \pm 36 \mu \mathrm{m}$ in $18 \mathrm{~mW} / \mathrm{cm}^{2}$ for the 5-minute group, and $201 \pm 82 \mu \mathrm{m}$ in $30 \mathrm{~mW} / \mathrm{cm}^{2}$ for the 3-minute group. A deeper and well-defined DL was found in the $3 \mathrm{~mW} / \mathrm{cm}^{2}$ and $9 \mathrm{~mW} / \mathrm{cm}^{2}$ groups. However, a patchy and an incomplete DL was noted in the higher intensity groups.

Bunsen-Roscoe law may be valid for the certain dose range, meaning that corneal cross-linking effect may depend on a threshold that the increase in the illumination intensity is limited up to $40-45 \mathrm{~mW}$ and the decrease in the illumination time is limited from 30 minutes to 2 minutes.

However, no statistically significant increase can be achieved for higher intensities ranging from $50 \mathrm{~mW}$ to $90 \mathrm{~mW}$ (illumination times of $<2$ minutes) probably due to complex biochemistry that is not completely understood until now. As the anterior stroma is more important in terms of biomechanical stability of the cornea, despite a significantly less DL depths after accelerated CXL, the cross-linking effect may also be strong enough to stop the progression of corneal ectasia which however must be validated by future long-term clinical studies. ${ }^{58}$

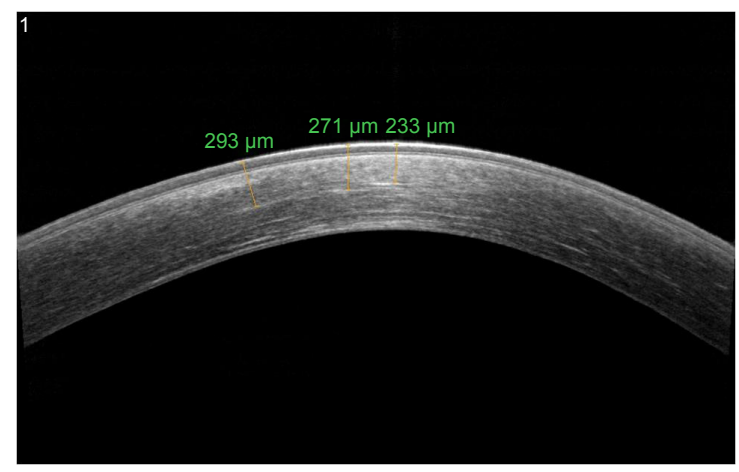

Figure 3 AS-OCT of keratoconus eye 6 months after accelerated epi-off corneal cross-linking radiating with UV-A at $10 \mathrm{~mW} / \mathrm{cm}^{2}$.

Note: $\mathrm{DL}$ is visible to a depth of $290 \mu \mathrm{m}$.

Abbreviations: AS-OCT, anterior segment optical coherence tomography; DL, demarcation line; epi-off, epithelium-off; UV-A, ultraviolet A.
Kymionis et $\mathrm{al}^{56}$ suggested that Bunsen-Roscoe law of reciprocity may not directly apply to CXL in living corneal tissue; thus, an increased total energy dose should probably be applied to the keratoconic cornea to achieve a treatment effect comparable with the already proven effective standard Dresden CXL protocol.

A study using confocal microscopy and AS-OCT after epi-off pulsed light (1 second on/1 second off) accelerated collagen cross-linking (PL-ACXL) and epi-off continuous light accelerated collagen cross-linking (CL-ACXL) evidenced a deeper DL in the PL-ACXL group. This could be probably due to an additional oxygen re-diffusion during pauses allowing more singlet oxygen release for the crosslinking of collagen molecules. ${ }^{59}$ Moramarco et al ${ }^{50}$ showed a mean depth of DL of $149 \mu \mathrm{m}$ in the CL-ACXL group and $213 \mu \mathrm{m}$ in the PL-ACXL group noting the importance of performing AS-OCT no later than 1 month after CXL to obtain the most accurate measure of the depth of DL.

Oxygen is essential for the photochemical polymerization reaction in CXL and is probably the limiting factor; it is theoretically possible that increasing fluence in an attempt to accelerate photopolymerization will not allow sufficient time for oxygen to diffuse and participate in the reaction, limiting the procedure. ${ }^{60}$

\section{DL in transepithelial epi-on CXL}

Transepithelial CXL with substances such as benzalkonium chloride, ethylenediaminetetraacetic acid, and trometamol to enhance the epithelial permeability of riboflavin has been developed as an alternative technique for thin corneas. ${ }^{25} \mathrm{In}$ epi-on CXL, OCT analysis after 2 weeks of CXL treatment shows a dense area in the corneal stroma (not present before CXL) that was linear in shape and positioned $\sim 100 \mu \mathrm{m}$ from the corneal epithelial layer, slightly beneath Bowman's membrane (Figure 4). These observations appear to highlight the possible role of the epithelium (also containing riboflavin) in shielding UV light. ${ }^{61}$ It is unclear whether the shallower DL using the epi-on approach is due to the limited penetration of riboflavin into the stroma, or it is a result of reduced UV-A light penetration by shielding from riboflavin-impregnated intact corneal epithelium. ${ }^{62}$

Bottos et $\mathrm{a}^{63}$ recognized the cause of different corneal stromal DL depth in decreased penetration of riboflavin into the stroma rather than the result of reduced UV-A light penetration through epithelium barrier. Furthermore, biomechanical effects of corneal CXL are closely related to oxygen supply, and the intact epithelium acts as a barrier to rapid oxygen diffusion into the corneal stroma and results 


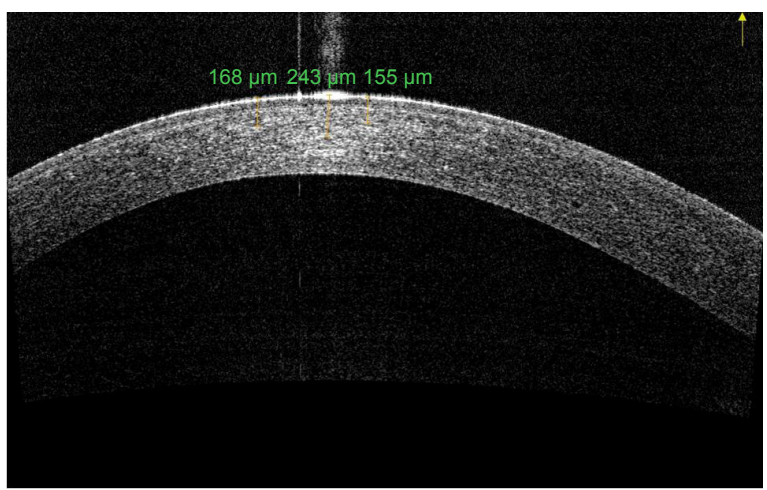

Figure 4 AS-OCT of keratoconus eye 6 months after epi-on corneal cross-linking radiating with UV-A at $3 \mathrm{~mW} / \mathrm{cm}^{2}$.

Note: $\mathrm{DL}$ is fairly visible to a depth of $180 \mu \mathrm{m}$

Abbreviations: AS-OCT, anterior segment optical coherence tomography; DL, demarcation line; epi-on, epithelium-on; UV-A, ultraviolet A.

in suboptimal cross-linking. These findings underscore, at a clinical level, the importance of corneal tissue oxygenation for effective CXL, as suggested by Richoz et al. ${ }^{60}$

\section{DL in transepithelial epi-on iontophoresis CXL}

I-CXL, when compared with transepithelial CXL, increases the penetration of riboflavin through intact epithelium enhancing the stromal amount of riboflavin while reporting lower photosensitizer amounts rather than standard epi-off protocols. ${ }^{32}$

I-CXL creates a DL that can be visualized with AS-OCT, which seems less easily distinguishable and shallower than in conventional CXL, with a particular OCT appearance (Figure 5). However, its depth and visualization seem to be more similar to conventional CXL than transepithelial CXL. ${ }^{64}$ Confocal microscopy noted loss of keratocytes

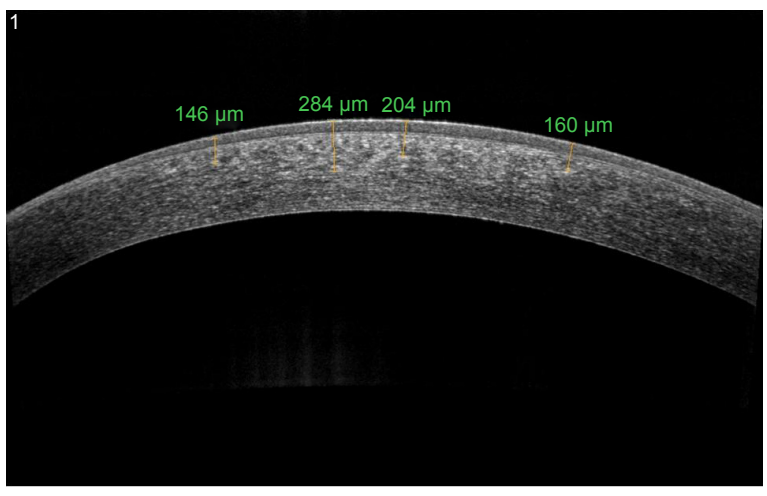

Figure 5 AS-OCT of keratoconus eye 6 months after epi-on corneal cross-linking with iontophoresis imbibition radiating with UV-A at $10 \mathrm{~mW} / \mathrm{cm}^{2}$.

Note: DL is barely discernible to a depth of $280 \mu \mathrm{m}$.

Abbreviations: AS-OCT, anterior segment optical coherence tomography; DL, demarcation line; epi-on, epithelium-on; UV-A, ultraviolet A. in the anterior and intermediate corneal stroma. DL was detected, $\sim 150-210 \mu \mathrm{m}$, in the I-CXL group with a characteristic pattern of "honeycomb" structure, which was less homogeneous compared with standard CXL. Bikbova and Bikbov $^{65}$ evidenced that a DL was not clearly measurable over time in patients who underwent I-CXL. They showed that a DL was observed in only $45 \%$ patients 1 month after transepithelial I-CXL and was completely absent in all these patients for 3 months post CXL. Vinciguerra et al ${ }^{32}$ described that, as in I-CXL, it is possible to detect an increase in reflectance in AS-OCT scans; however, no DL was visible and this finding could be explained by either the different concentration gradient induced by iontophoresis or a reduced CXL effect. Zhang et $\mathrm{al}^{66}$ showed that the epithelial cells are not enriched with riboflavin. For this reason, only a small part of the UV light should be absorbed by the epithelium $(\sim 15 \%-20 \%)$.

\section{Conclusion}

Corneal cross-link is a strategy based on the underlying pathology of the corneal ectatic diseases. After corneal CXL procedures, a stromal DL can be observed within the corneal stroma at 1 month postoperatively. It would be unreasonable to disagree with the interpretation of the depth of DL as an indirect measurement of CXL penetration within the stroma, but the current knowledge suggests that the "the deeper, the better" principle is rather a simplistic approach for interpreting the clinical importance of the corneal stromal DL.

We can conclude that there is still much to understand about the changes of corneal structure after the photochemical CXL reaction. Experimental and clinical researchers on CXL have proven a good effectiveness in stabilizing keratoconus and iatrogenic keratectasia. Moreover, the constant aim of basic and clinical research will be the identification of the best strategies of treatment that is able to obtain the best clinical efficacy together with the maximum safety profile with very few side effects.

\section{Disclosure}

The authors report no conflicts of interest in this work.

\section{References}

1. Wollensak G, Spoerl E, Seiler T. Riboflavin/ultraviolet-A-induced collagen crosslinking for the treatment of keratoconus. Am J Ophthalmol. 2003;135(5):620-627.

2. Spadea L. Corneal collagen cross-linking with riboflavin and UV-A irradiation in pellucid marginal degeneration. J Refract Surg. 2010; 26(5):375-377.

3. Ziaei M, Barsam A, Shamie N, et al; ASCRS Cornea Clinical Committee. Reshaping procedures for the surgical management of corneal ectasia. J Cataract Refract Surg. 2015;41(4):842-872. 
4. Gordon-Shaag A, Millodot M, Shneor E, Liu Y. The genetic and environmental factors for keratoconus. Biomed Res Int. 2015;2015:795738.

5. Binder PS. Analysis of ectasia after laser in situ keratomileusis: risk factors. J Cataract Refract Surg. 2007;33(9):1530-1538.

6. Spadea L, Cantera E, Cortes M, Conocchia NE, Stewart CW. Corneal ectasia after myopic laser in situ keratomileusis: a long-term study. Clin Ophthalmol. 2012;6:1801-1813.

7. Kirwan C, O'Malley D, O'Keefe M. Corneal hysteresis and corneal resistance factor in keratoectasia: finding using the Reichert ocular response analyzer. Ophthalmologica. 2008;222(5):334-337.

8. Vazirani J, Basu S. Keratoconus: current perspectives. Clin Ophthalmol. 2013;7:2019-2030.

9. O’Brart DP. Corneal collagen cross-linking: a review. J Optom. 2014; 7(3):113-124.

10. Hammerstein W. Zur genetic des keratoconus [The genetics of keratoconus]. Albrecht Von Graefes Arch Klin Exp Ophthalmol. 1974;190(4):293-308.

11. Zhou L, Sawaguchi S, Twining SS, Sugar J, Feder RS, Yue BY. Expression of degradative enzymes and protease inhibitors in corneas with keratoconus. Invest Ophthalmol Vis Sci. 1998;39(7):1117-1124.

12. Andreassen T, Simonsen AH, Oxlund H. Biomechanical properties of keratoconus and normal corneas. Exp Eye Res. 1980;31(4):435-441.

13. Kuo IC, Broman A, Pirouzmanesh A, Melia M. Is there an association between diabetes and keratoconus. Ophthalmology. 2006;113(2): 184-190.

14. Cannon DJ, Foster CS. Collagen crosslinking in keratoconus. Invest Ophthalmol Vis Sci. 1978;17(1):63-65.

15. Spadea L, Maraone G, Cagini C. A case of unilateral circumscribed posterior keratoconus evaluated by three different imaging tools: optical coherence tomography, videokeratography, and Scheimpflug corneal tomography. Int Ophthalmol. 1-5. Epub May 13, 2016.

16. Panos GD, Hafezi F, Gatzioufas Z. Pellucid marginal degeneration and keratoconus; differential diagnosis by corneal topography. $J$ Cataract Refract Surg. 2013;39(6):968.

17. Spoerl E, Huhle M, Seiler T. Induction of cross-links in corneal tissue Exp Eye Res. 1998;66(1):97-103.

18. Raiskup-Wolf F, Hoyer A, Spoerl E, Pillunat LE. Collagen crosslinking with riboflavin and ultraviolet-A light in keratoconus: long-term results. J Cataract Refract Surg. 2008;34(5):796-801.

19. Caporossi A, Mazzotta C, Baiocchi S, Caporossi T. Long-term results of riboflavin ultraviolet A corneal collagen crosslinking for keratoconus in Italy: the Siena eye cross study. Am J Ophthalmol. 2010; 149(4):585-593.

20. Wittig-Silva C, Whiting M, Lamoureux E, Lindsay RG, Sullivan LJ, Snibson GR. A randomized controlled trial of corneal collagen crosslinking in progressive keratoconus: preliminary results. J Refract Surg. 2008;24(7):S720-S725.

21. Caporossi A, Mazzotta C, Baiocchi S, Caporossi T, Denaro R. Agerelated long-term functional results after riboflavin UV A corneal crosslinking. J Ophthalmol. 2011;2011:608041.

22. Spoerl E, Seiler T. Techniques for stiffening the cornea. J Refract Surg. 1999;15(6):711-713.

23. Randleman JB, Khandelwal SS, Hafezi F. Corneal cross-linking. Surv Ophthalmol. 2015;60(6):509-523.

24. Spadea L, Salvatore S, Paroli MP, Vingolo EM. Recovery of corneal sensitivity after collagen crosslinking with and without epithelial debridement in eyes with keratoconus. J Cataract Refract Surg. 2015;41(3):527-532.

25. Spadea L, Mencucci R. Transepithelial corneal collagen cross-linking in ultrathin keratoconic corneas. Clin Ophthalmol. 2012;6:1785-1792.

26. Gatzioufas Z, Richoz O, Brugnoli E, Hafezi F. Safety profile of high-fluence corneal collagen cross-linking for progressive keratoconus: preliminary results from a prospective cohort study. J Refract Surg. 2013;29(2):846-848.

27. Beshtawi IM, Akhtar R, Hillarby MC, et al. Biomechanical changes after repeated collagen cross-linking on human corneas assessed in vitro using scanning acoustic microscopy. Invest Ophthalmol Vis Sci. 2014; 55(3):1549-1554.

28. Raiskup F, Spoerl E. Corneal crosslinking with riboflavin and ultraviolet A. I. Principles. Ocul Surf. 2013;11(2):65-74.
29. Mastropasqua L, Lanzini M, Curcio C, et al. Structural modifications and tissue response after standard epi-off and iontophoretic corneal crosslinking with different irradiation procedures. Invest Ophthalmol Vis Sci. 2014;55(4):2526-2533.

30. Mencucci R, Ambrosini S, Paladini I, et al. Early effects of corneal collagen cross-linking by iontophoresis in ex vivo human corneas. Graefes Arch Clin Exp Ophthalmol. 2015;253(2):277-286.

31. Bikbova G, Bikbov M. Transepithelial corneal collagen cross-linking by iontophoresis of riboflavin. Acta Ophthalmol. 2014;92(1):30-34.

32. Vinciguerra P, Randleman JB, Romano V, et al. Transepithelial iontophoresis corneal collagen cross-linking for progressive keratoconus: initial clinical outcomes. J Refract Surg. 2014;30(11):746-753.

33. Kanellopoulos AJ, Binder PS. Management of corneal ectasia after LASIK with combined, same-day, topography-guided partial transepithelial PRK and collagen cross-linking: the Athens protocol. $J$ Refract Surg. 2011;27(5):323-331.

34. Kymionis GD, Portaliou DM, Diakonis VF, et al. Management of post laser in situ keratomileusis ectasia with simultaneous topography guided photorefractive keratectomy and collagen cross-linking. Open Ophthalmol J. 2011;5:11-13.

35. Spadea L, Paroli M. Simultaneous topography-guided PRK followed by corneal collagen cross-linking after lamellar keratoplasty for keratoconus. Clin Ophthalmol. 2012;6:1793-1800.

36. Kymionis GD, Portaliou DM, Diakonis VF, et al. Posterior linear stromal haze formation after simultaneous photorefractive keratectomy followed by corneal collagen cross-linking. Invest Ophthalmol Vis Sci. 2010; 51(10):5030-5033.

37. Ertan A, Karacal H, Kamburoglu G. Refractive and topographic results of transepithelial cross-linking treatment in eyes with intacs. Cornea. 2009;28(7):719-723.

38. Renesto Ada C, Melo LA Jr, Sartori Mde F, Campos M. Sequential topical riboflavin with or without ultraviolet a radiation with delayed intracorneal ring segment insertion for keratoconus. Am J Ophthalmol. 2012;153(5):982-993.

39. Spadea L, Maraone G, Cagini C. Sliding keratoplasty followed by transepithelial iontophoresis collagen cross-linking for pellucid marginal degeneration. J Refract Surg. 2016;32(1):47-50.

40. Kanellopoulos AJ. Long-term safety and efficacy follow-up of prophylactic higher fluence collagen cross-linking in high myopic laser-assisted in situ keratomileusis. Clin Ophthalmol. 2012;6:1125-1130.

41. Croxatto JO, Tytiun AE, Argento CJ. Sequential in vivo confocal microscopy study of corneal wound healing after crosslinking in patients with keratoconus. J Refract Surg. 2010;26(9): 638-645.

42. Mazzotta C, Balestrazzi A, Traversi C, et al. Treatment of progressive keratoconus by riboflavin UVA induced cross linking of corneal collagen: ultrastructural analysis by Heidelberg retinal tomography II in vivo confocal microscopy in humans. Cornea. 2007;26(4): 390-397.

43. Mazzotta C, Balestrazzi A, Baiocchi S, Traversi C, Caporossi A. Stromal haze after combined riboflavin-UVA corneal collagen cross-linking in keratoconus: in vivo confocal microscopic evaluation. Clin Experiment Ophthalmol. 2007;35(6):580-582.

44. Dhaliwal JS, Kaufman SC. Corneal collagen cross-linking: a confocal, electron, and light microscopy study of eye bank corneas. Cornea. 2009; 28(1):62-67.

45. Kamaev P, Friedman MD, Sherr E, Muller D. Photochemical kinetics of corneal cross-linking with riboflavin. Invest Ophthalmol Vis Sci. 2012;53(4):2360-2367.

46. Wollensak G, Spoerl E, Wilsch M, Seiler T. Keratocyte apoptosis after corneal collagen cross-linking using riboflavin/UVA treatment. Cornea. 2004;23(1):43-49.

47. Seiler T, Hafezi F. Corneal cross-linking-induced stromal demarcation line. Cornea. 2006;25(9):1057-1059.

48. Kymionis GD, Tsoulnaras KI, Liakopoulos DA, Skatharoudi CA, Grentzelos MA, Tsakalis NG. Corneal stromal demarcation line depth following standard and a modified high intensity corneal cross-linking protocol. J Refract Surg. 2016;32(4):218-222. 
49. Yam JC, Chan CW, Cheng AC. Corneal collagen cross-linking demarcation line depth assessed by Visante OCT after CXL for keratoconus and corneal ectasia. J Refract Surg. 2012;28(7):475-481.

50. Moramarco A, Iovieno A, Sartori A, Fontana L. Corneal stromal demarcation line after accelerated crosslinking using continuous and pulsed light. J Cataract Refract Surg. 2015;41(11):2546-2551.

51. Doors M, Tahzib NG, Eggink FA, Berendschot TT, Webers CA, Nuijts RM. Use of anterior segment optical coherence tomography to study corneal changes after collagen cross-linking. Am J Ophthalmol. 2009;148(6):844-851.

52. Gatzioufas Z, Balidis M, Kozeis N. Is the corneal stromal demarcation line depth a true indicator of corneal collagen crosslinking efficacy? J Cataract Refract Surg. 2016;42(5):804.

53. Kymionis GD, Grentzelos MA, Plaka AD, et al. Correlation of the corneal collagen cross-linking demarcation line using confocal microscopy and anterior segment optical coherence tomography in keratoconic patients. Am J Ophthalmol. 2014;157(1):110-115.

54. Malta JB, Renesto AC, Moscovici BK, Soong HK, Campos M. Stromal demarcation line induced by corneal cross-linking in eyes with keratoconus and non keratoconic asymmetric topography. Cornea. 2015; 34(2):199-203.

55. Kymionis GD, Tsoulnaras KI, Grentzelos MA, et al. Evaluation of corneal stromal demarcation line depth following standard and a modifiedaccelerated collagen cross-linking protocol. Am J Ophthalmol. 2014; 158(4):671-675.

56. Kymionis GD, Tsoulnaras KI, Grentzelos MA, et al. Corneal stroma demarcation line after standard and high-intensity collagen crosslinking determined with anterior segment optical coherence tomography. $J$ Cataract Refract Surg. 2014;40(5):736-740.

57. Shetty R, Pahuja NK, Nuijts RM, et al. Current protocols of corneal collagen crosslinking - visual, refractive and tomographic outcomes. Am J Ophthalmol. 2015;160(2):243-249.
58. Ozgurhan EB, Sezgin Akcay BI, Yildirim Y, Karatas G, Kurt T, Demirok A. Evaluation of corneal stromal demarcation line after two different protocols of accelerated corneal collagen cross-linking procedures using anterior segment optical coherence tomography and confocal microscopy. J Ophthalmol. 2014;2014:981893.

59. Mazzotta C, Traversi C, Caragiuli S, Rechichi M. Pulsed vs continuous light accelerated corneal collagen crosslinking: in vivo qualitative investigation by confocal microscopy and corneal OCT. Eye (Lond). 2014; 28(10):1179-1183.

60. Richoz O, Hammer A, Tabibian D, Gatzioufas Z, Hafezi F. The biomechanical effect of corneal collagen cross-linking (CXL) with riboflavin and UV-A is oxygen dependent. Transl Vis Sci Technol. 2013;2(7):6.

61. Filippello M, Stagni E, O’Brart D. Transepithelial corneal collagen crosslinking: bilateral study. J Cataract Refract Surg. 2012;38(2): 283-291.

62. Chen X, Stojanovic A, Eidet JR, Utheim TP. Corneal collagen crosslinking (CXL) in thin corneas. Eye Vis (Lond). 2015;2:15.

63. Bottos KM, Schor P, Dreyfuss JL, Nader HB, Chamon W. Effect of corneal epithelium on ultraviolet-A and riboflavin absorption. Arq Bras Oftalmol. 2011;74(5):348-351.

64. Bonnel S, Berguiga M, De Rivoyre B, et al. Demarcation line evaluation of iontophoresis-assisted transepithelial corneal collagen cross-linking for keratoconus. J Refract Surg. 2015;31(1):36-40.

65. Bikbova G, Bikbov M. Standard corneal collagen crosslinking versus transepithelial iontophoresis-assisted corneal crosslinking, 24 months follow-up: randomized control trial. Acta Ophthalmol. Epub 2016 Apr 4

66. Zhang Y, Sukthankar P, Tomich JM, Conrad GW. Effect of the synthetic NC-1059 peptide on diffusion of riboflavin across an intact corneal epithelium. Invest Ophthalmol Vis Sci. 2012;53(6):2620-2629.
Clinical Ophthalmology

\section{Publish your work in this journal}

Clinical Ophthalmology is an international, peer-reviewed journal covering all subspecialties within ophthalmology. Key topics include: Optometry; Visual science; Pharmacology and drug therapy in eye diseases; Basic Sciences; Primary and Secondary eye care; Patient Safety and Quality of Care Improvements. This journal is indexed on Submit your manuscript here: http://www.dovepress.com/clinical-ophthalmology-journal

\section{Dovepress}

PubMed Central and CAS, and is the official journal of The Society of Clinical Ophthalmology (SCO). The manuscript management system is completely online and includes a very quick and fair peer-review system, which is all easy to use. Visit http://www.dovepress.com/ testimonials.php to read real quotes from published authors. 